\title{
Wear recording at micro deep drawing tools with comparative digital holography
}

\author{
S. Huferath-von Luepke \\ huferath@bias.de \\ P. Huke \\ C. von Kopylow \\ R. B. Bergmann
}

\author{
Bremer Institut für angewandte Strahltechnik - BIAS GmbH, Klagenfurter Str. 2, 28359 Bremen, Ger- \\ many \\ Bremer Institut für angewandte Strahltechnik - BIAS GmbH, Klagenfurter Str. 2, 28359 Bremen, Ger- \\ many \\ Bremer Institut für angewandte Strahltechnik - BIAS GmbH, Klagenfurter Str. 2, 28359 Bremen, Ger- \\ many \\ Bremer Institut für angewandte Strahltechnik - BIAS GmbH, Klagenfurter Str. 2, 28359 Bremen, Ger- \\ many
}

We present a method to record the wear on samples of micro deep drawing tools. The method is based on the basic idea of comparative holography and captures three dimensionally the surface of the tool in the initial state and compares it with used state after processing several parts. The comparison reveals scratches and other effects caused by wear. In an industrial environment the comparative method must compensate for linear and rotational displacement of the present tool with respect to the initial state. As a consequence to the single-step-measurement with sufficiently high illumination intensity the developed set-up can be made insusceptible against stray light and the method is capable of inline quality control during a production process in a micro deep drawing machine.

[DOI: http://dx.doi.org/10.2971/jeos.2012.12041]

Keywords: Comparative digital holography, micro tools, wear detection

\section{INTRODUCTION}

In the deep drawing process of micro parts, tribological effects play a far more significant role than in macro processes [1, 2]. In order to minimize effects, research focuses on enhanced tools manufactured from friction-reduced materials and/or with friction-reducing micro structures upon the surfaces $[3,4]$. A measuring technique which measures wear at micro deep drawing tools has to be introduced. Wear occurs mainly on surfaces under high loading, for example on the top face and drawn radius of a micro deep drawing die. A fast measurement technique is preferable to avoid the influence of vibrations and to keep the downtime of the machine as short as possible. Furthermore, a large aperture is necessary due to small lateral dimensions of the tool and the wear. With regard to the vertical dimension of the tool an enlarged depth of focus is advantageous.

Friction is often reduced by structuring critical surfaces. The size of these structures is in the $\mu \mathrm{m}$-range and may produce additional noise for a sensitive measurement technique. Therefore, the used tool has to be compared with its initial state in order to figure out if the measured structure is unaltered or worn. Finally, a measurement technique which can be used inline is advantageous, since removing and inserting tools is not convenient for an industrial process.

There are many measurement techniques that can be used for surface, micro and nano metrology $[5,6]$. They can be subdivided into stylus and optical based probes. In Whitehouse [5] Section 4 a detailed description of different stylus and optical based probes is given. Whitehouse [5] as well as Bergmann et al. [7] discuss the advantages and disadvantages of stylus and optical based probes. The main information we obtain from these comparisons is that the stylus based probes may damage the tool. This is particularly crucial for micro-tools, which are sensitive to every contact. The techniques also can be subdivided into their type of sampling [8]: 1D (point sensor), 2D (line sensor) and 3D (area sensor) and / or into scanning or non-scanning methods. 1D, 2D and scanning techniques like atomic force microscopy [9], tactile coordinate measuring [10], triangulation (1D or 2D) [11, 12], white light interferometry [13], confocal microscopy [14, 15] - to name just a few - have high resolution and would fulfil the industrial requirements regarding the resolution of the measuring system. However, 1D and 2D systems as well as scanning systems are not feasible because their measurement times exceed our given time restriction. Furthermore, vibrations cause misalignment between the object and the measuring system. This may cause deviations and misinterpretations during measurement. Therefore 3D techniques like micro fringe reflection [16], shearography [17], phase retrieval [18], interferometry [19] and digital holography [19] seem more useful for the industrial application we are interested in. All these techniques need only a few measurements to capture the shape of the object. Bergmann et al. [20] give a good 
overview of non-destructive techniques especially about the applications of micro fringe reflection, shearography. Agour et al. [21] illustrate that phase retrieval is an adequate and fast technique to measure micro parts under lab conditions where only four images have to be taken. Falldorf et al. [22] illustrate that digital holography is a suitable technique to measure the deformation of micro switches caused by heating. They compared their results with an interferometric set-up and showed that digital holography is the best technique for the investigated object. Furthermore digital holography is a technique which allows for capturing the wave field of an object with a single shot with an exposure time well below $1 \mathrm{sec}$. This is important for inline measurements in an industrial environment where vibrations complicate measurements. Due to the wavelength being in the $0.5 \mu \mathrm{m}$ range, the phase of the measurement is not unambiguously determined and therewith no statement of the height can be made. Therefore two measurements at different wavelengths have to be carried out. The synthesis deliver a wavelength in the necessary range to capture an ambiguous phase. The real shape of the object can be derived from this phase. In our first experiments we use these two measurements, knowing that therewith the advantage of digital holography compared to the other $3 \mathrm{D}$ techniques is gone for the same reason more than one measurement. Later on the measurement with two wavelengths should be performed with a single shot to overcome this problem. Hansel et al. [23] had shown one solution for two wavelengths contouring with a single shot measurement by using a femtosecond laser.

Due to the above described points like being non-destructive, fast and able to measure contours with a single shot [23] we decided to use digital holography for the measurement of our tools. Particularly, we use comparative digital holography $(\mathrm{CDH})$ which is established in the macro range [24, 25]. $\mathrm{CDH}$ offers the possibility to compare the worn tool directly with its initial state by illuminating it with the wavefront of the initial state. Up to now $\mathrm{CDH}$ has not been adapted to the requirements of measurements of micro tools in an industrial environment.

In order to introduce $\mathrm{CDH}$ for the measurement of micro tools we first scale down the set-up and increase therewith the aperture. It has to be taken into account that the reference beam is the limiting factor for the set-up we use. Due to the lens less Fourier holography [19] set-up the reference source point has to be placed in the same plane as the object and close to the object to avoid aliasing. The spherical reference wave has a Gaussian profile and the minimal distance between camera and reference source point is restricted. Below this the CCD target will not be completely illuminated. For our arrangement the minimum distance is about $40 \mathrm{~mm}$. In a second step which is not part of this paper we have to implement a spatial light modulator (SLM).

In this paper we present the first results of a miniaturized holographic set-up for $\mathrm{CDH}$ and the comparison of these results with the commercially available confocal microscope from Keyence corp. We demonstrate that scratches deeper than $5 \mu \mathrm{m}$ are detectable with our set-up.

\section{HOLOGRAPHIC RECONSTRUCTION}

The reconstruction algorithm we use in this paper is based on Rayleigh's first integral [26]:

$$
b^{\prime}(x, y)=\frac{1}{i \lambda} \int_{-\infty}^{\infty} \int_{\infty} h(\xi, \eta) r^{\prime}(\xi, \eta) \frac{e^{i k \rho}}{\rho} \cos (\theta) d(\xi, \eta),
$$

with

$$
\rho=\sqrt{(x-\xi)^{2}+(y-\eta)^{2}+d^{2}} .
$$

Here, $(x, y)$ and $(\xi, \eta)$ are the coordinates of the object plane, image plane and of the hologram plane respectively, $b^{\prime}(x, y)$ is the reconstructed wave field, $h(\xi, \eta)$ the hologram, $r^{\prime}(\xi, \eta)$ the reference wave and $d$ the distance between object and hologram plane. To simplify Eq. (1) we apply the Fresnel approximation [26]. Due to the spherical reference wave which has its origin in the object plane the reference part can be eliminated [27]. This leads to the following equation:

$$
b^{\prime}(x, y)=S_{1}(x, y) \cdot \mathcal{F}^{-1}\{h(\xi, \eta)\}\left(\frac{x}{\lambda d}, \frac{y}{\lambda d}\right),
$$

with

$$
S_{1}(x, y)=\frac{1}{\lambda d} e^{-i \frac{2 \pi d}{\lambda}} e^{-i \frac{\pi}{\lambda d}|(x, y)|^{2}} .
$$

Where $\mathcal{F}^{-1}\{\ldots\}$ is the operator of the inverse two-dimensional Fourier transformation and $\lambda$ the wavelength [27].

In the Fresnel propagation (Eq. (3)) algorithm the Fourier transformation from one spatial domain into another depend on the propagation distance and the wavelength. As a consequence, different pixel sizes occur in the reconstruction plane for different wavelengths. This different pixel size leads to an enlargement of the reconstructed image of the larger wavelength compared to the one with the smaller wavelength. Therefore the pixel and therewith the complete hologram have to be rescaled by a certain, measurable factor. To rescale the hologram the scaling factor between the two pixel sizes within the reconstruction plane has to be determined and the hologram generated with the smaller wavelength is multiplied by this scaling factor to increase the overall size of the image. Now both holograms have the same pixel size within the same reconstruction plane but the reconstructions differ - which is corrected by introducing a rectangular aperture (rect.-function). Due to the different size we are not able to combine the two holograms directly. Thence the size of the images is matched by cutting the overlapping area. This step can be described as introducing a rectangular aperture which is mathematically described by a rect-function. Due to the fact that we have a Fourier transformation within the reconstruction algorithm we get an interaction with a sinc-function within the Fourier domain which is in our case the reconstruction plane. This can be seen in Eq. (5) [26]:

$$
\mathcal{F}\left\{\operatorname{rect}\left(\frac{\xi}{2 w_{x}}, \frac{\eta}{2 w_{y}}\right)\right\}=B \cdot \operatorname{sinc}\left(\frac{2 w_{x} x}{\lambda z}, \frac{2 w_{y} y}{\lambda z}\right),
$$




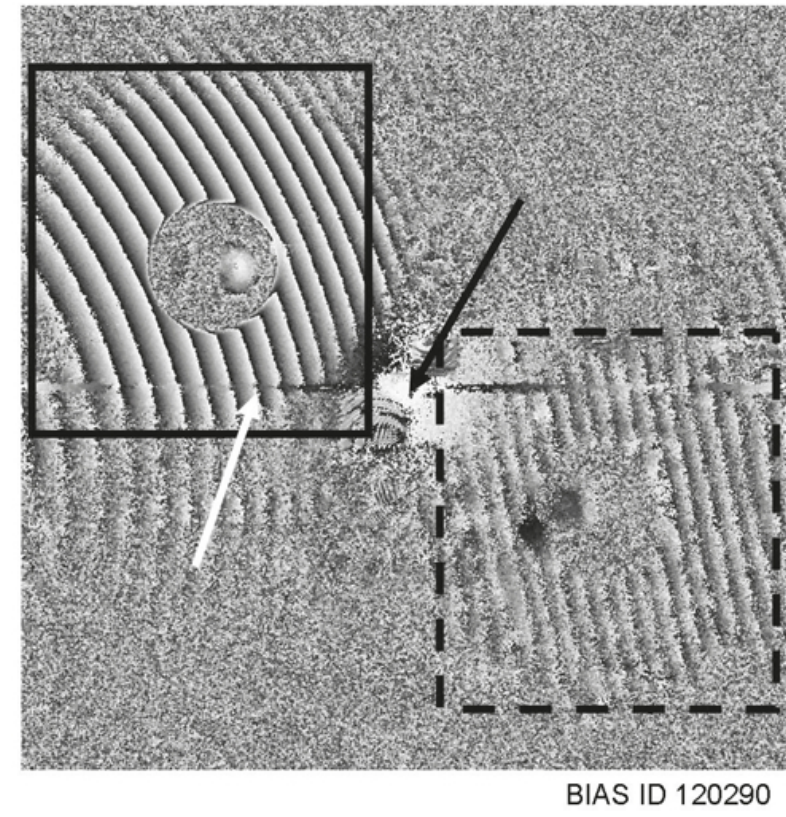

FIC. 1 Reconstructed phase difference of two holograms of the unused tool with different wavelengths, where the pixel size of one hologram is matched to the other. Hence, a pattern along the $x$-axis in the centre of the image appears (white arrow). Because a complete reconstruction is illustrated, the virtual orthoscopic (solid lined box) and the real pseudoscopic image (dashed box) and the zero order (black arrow) can be seen.

with $B=4 w_{x} w_{y}$. Here, $w_{x}$ and $w_{y}$ correspond to the half width of the aperture. Due to the aperture a pattern along the $x$ - and $y$-axis appears, because of the cutting of the overlapping area.

Figure 1 illustrates the pattern described above which can be clearly seen in the middle of the image along the x-axis (white arrow). Within the figure we see a reconstructed phase difference of an unused tool. The virtual orthoscopic image of the tool in the solid lined box will be explained later on in this paper while the zero order (black arrow) and the real pseudoscopic image (dashed box) will be cut out. The pattern (white arrow) may be suppressed be introducing a Hanning window [28].

\section{MEASURING SET-UP}

Figure 2 is a photograph of the deep drawing tool sample with die diameters ranging from 2.3 to $5 \mathrm{~mm}$. The image enlargement within Figure 2 shows the part of the surface of the sample with a die diameter of $2.2 \mathrm{~mm}$ and a drawn radius of $0.5 \mathrm{~mm}$. The latter is an interesting area we want to investigate. This image was taken with a confocal microscope from Keyence corp.. The horizontal mark which is clearly visible within the image enlargement is an artefact of the manufacturing process and not part of the investigation.

The sample illustrated in the image enlargement in Figure 2 was used for measurement of the initial and worn state. After measuring the initial state of this sample we simulated wear upon its surface by introducing scratches with a depth of up to $30 \mu \mathrm{m}$.

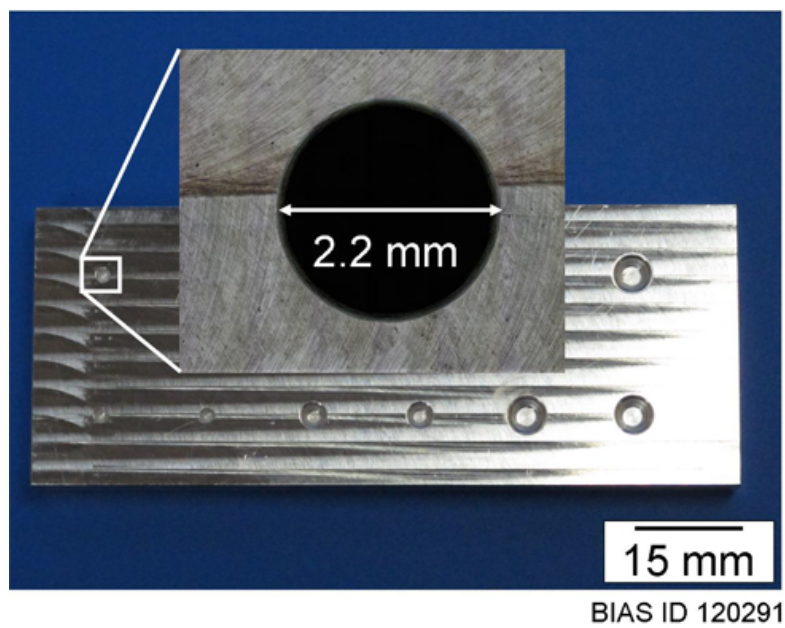

FIG. 2 Photograph of different deep drawing tools. The image enlargement illustrates a sample with a die diameter of $2.2 \mathrm{~mm}$ and a drawn radius of $0.5 \mathrm{~mm}$ taken by a confocal microscope. The horizontal mark within the enlargement is an artefact of the manufacturing process and not part of the investigation.

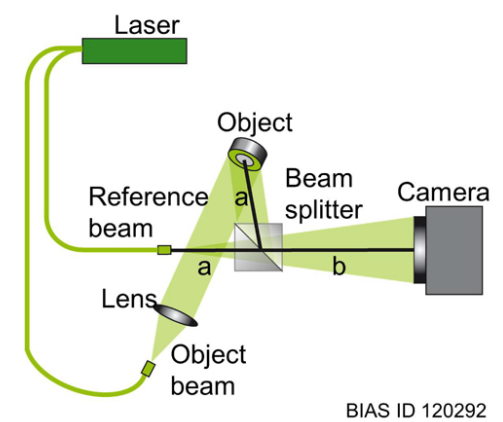

FIG. 3 Schematic sketch of the set-up used to capture the holograms of the initial and worn state of the tool. The distance $a$ from the centre to the beam splitter to either the object and the reference source point equals. The total distance $a+b$ between reference source point and camera respectively object and camera is $75 \mathrm{~mm}$.

Figure 3 is a schematic sketch of the set-up we used to capture the initial and worn state of our sample. The set-up is a lens less Fourier holography set-up. Hence, the source point of the reference wave is in the same plane as the object. With regard to the size of our sample, it is not possible to place the reference source point close enough to the part of the sample we investigate using a conventional set-up as it is necessary to avoid aliasing.

Figure 3 illustrates our set-up, that overcomes the problem described above by introducing a beam splitter into the set-up. Via this beam splitter the reference beam is guided into the set-up by placing the source in the same distance from the beam splitter as the object. Due to the size of the beam splitter we could not maintain the minimal distance of $40 \mathrm{~mm}$ between object and camera which was mentioned in Section 2. The total distance between reference source point and camera respectively object and camera is $a+b=75 \mathrm{~mm}$. 

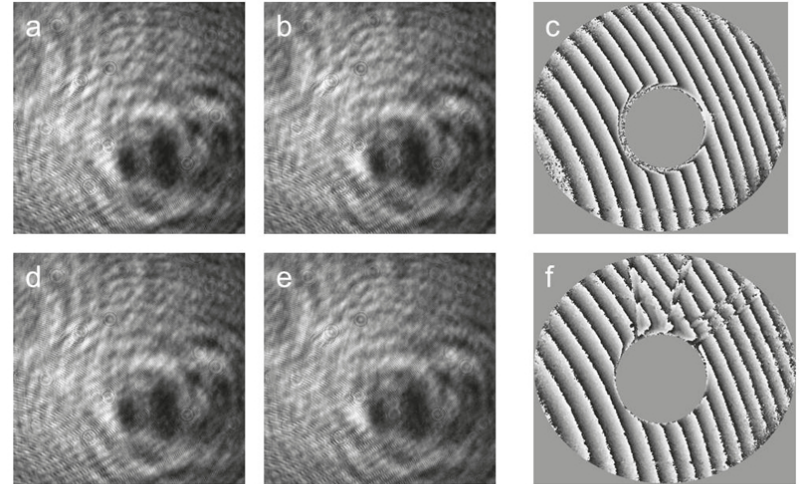

BIAS ID 120293

FIC. 4 Hologram of the initial state of the tool at a) $\lambda_{1}=575.113 \mathrm{~nm}$ and b) $\lambda_{2}=577.621 \mathrm{~nm}$; c) reconstruction of the masked phase difference from a) and b) and hologram of the worn state of the tool at d) $\lambda_{1}=575.113 \mathrm{~nm}$ and e) $\lambda_{2}=577.621 \mathrm{~nm} ; \mathrm{f}$ ) reconstruction of the masked phase difference from $\mathrm{d}$ ) and e). The disruptions in the fringe pattern of the upper part of $f$ ) are scratches which have to be investigated while the horizontal mark seen in the lower part of $c$ ) and f) are reconstruction artefacts.

\section{GENERATION OF THE STATE DIFFERENCE}

We used a tunable dye laser with a wavelength range between 560 and $580 \mathrm{~nm}$. The holograms were taken with the set-up described above and the two wavelengths were $\lambda_{1}=575.113 \mathrm{~nm}$ and $\lambda_{2}=577.621 \mathrm{~nm}$, respectively. The resulting synthetic wavelength $\Lambda$ is given by

$$
\Lambda=\frac{\lambda_{1} \cdot \lambda_{2}}{\left|\lambda_{1}-\lambda_{2}\right|}
$$

is $132.46 \mu \mathrm{m}$. Therefore, the maximal height of one fringe $(2 \pi)$ can be calculated by using

$$
\Delta \phi=\frac{2 \pi}{\Lambda} S
$$

with

$$
S=2 \Delta h .
$$

Where $\Delta \phi$ describes the phase and $S$ the optical phase difference which is twice the height $h$ (Eq. (8)). By means of these equations we know that a height difference below $\Delta h=66.23 \mu \mathrm{m}$ can be determined unambiguously.

Figure 4(a) and 4(b) show two holograms of the initial state of the tool captured with two different wavelengths. The resulting phase difference is shown in Figure 4(c). For a better illustration a masked part of the reconstructed, virtual orthoscopic part is displayed. The high number of fringes within the phase difference is due to the capturing arrangement where the object is tilted. The horizontal disruption of the fringe pattern in the lower part of the phase difference, visible e.g. in Figures $4(\mathrm{c})$ or $4(\mathrm{f})$, from the reconstruction algorithm as mentioned in Section 2.

Figure 4(d) and 4(e) show two holograms of the worn state of the tool and Figure 4(f) the resulting masked and cut
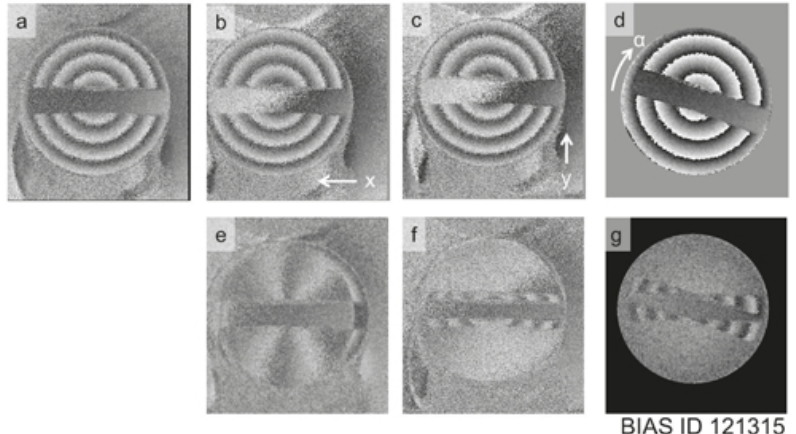

FIG. 5 a) phase difference of the master (new) object; b), c) and d) misaligned test (worn) objects. The misalignment takes place by a shift in $\mathrm{x}$ - (b) or y-direction (c) or by rotation (d). e), f) and $g$ ) show the state difference of the master and the misaligned test object.

phase difference. Within the upper part of the phase difference scratches of the worn tool are clearly visible. This indicates that the method produces sufficient information about the wear.

To simulate the $\mathrm{CDH}$ condition, which means that the used tool is illuminated by its initial state using a SLM, we merge the holograms of the initial and the worn state by subtracting them. For calculating the state difference we have to take into account that the sample was removed for generating the wear. Hence, the position of the tool may not be the same as before. This means that the worn tool may be shifted, rotated and / or tilted. The consequence of a displacement are additional fringes [19] within the state difference. To get a meaningful result about the wear this displacement has to be corrected. Figure 6(b) illustrates a state difference where the misalignment was corrected. In this figure only the change of the two states play a role. Another important point is, more scratches are noticeable than within the phase difference of the worn tool itself (Figure 4(f)).

\section{MISALIGNMENT CORRECTION}

If misalignment like shift of the worn object compared to the measurement of its initial state takes place additional fringes appear which lead to misinterpretations of the resulting image. Figure 5 illustrates how misalignment affects the state difference between new and worn tool. For better visualisation we chose a screw head as master (new) and test (worn) object. Figure 5(a) shows the phase difference of the master object while (b), (c) and (d) illustrate the phase differences of a test object which is shifted in $\mathrm{x}$-direction, shifted in y-direction and rotated respectively. Due to this misalignment of the test object compared to the master, fringes appear within the state difference of master and test. This is shown in Figure 5(e,f,g).

It can be assumed that these fringes are caused by an additional phase ramp. The reason for this assumption is that a phase ramp in the frequency domain - here the hologram plane - leads to a shift in the spatial domain - here the object plane [26]. For the correction of the misalignment in $x$ - and $y-$ direction we used the shift theorem [29] as it is described in 

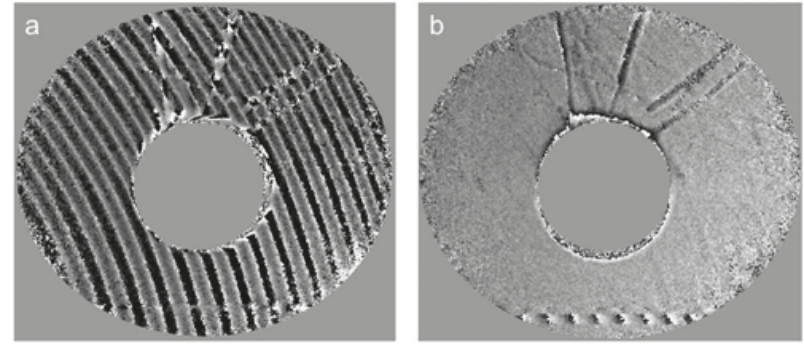

BIAS ID 120294

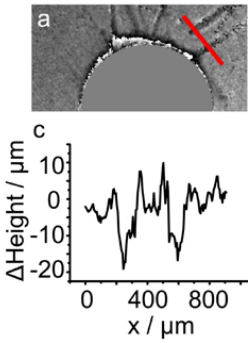

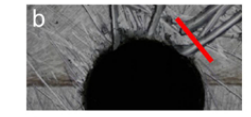

$\varepsilon^{\mathrm{d}}$

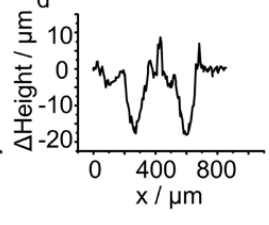

BIAS ID 120295
FIG. 6 State difference of the tool with a) uncorrected shift and b) corrected shift.

Eq. (9) [27]:

$$
\begin{aligned}
\arg \left[b_{i}^{\prime}(x, y)\right]= & \arg \left[S_{1 i}\right]+\Delta \phi_{B i}(\xi, \eta)+\phi_{0 i} \\
& +\arg \left[\mathcal{F}^{-1}\left\{h_{i}(\xi, \eta)\right\}\right]\left(\frac{x}{\lambda d}, \frac{y}{\lambda d}\right) .
\end{aligned}
$$

Where $\Delta \phi_{B i}(\xi, \eta)$ describes the phase ramp which introduces the shift, $\phi_{0 i}$ is a constant phase term and $i=1,2$ stands for the two different wavelengths. This equation contains the general part of back propagating a hologram $\left(\mathcal{F}^{-1}\left\{h_{i}(\xi, \eta)\right\}\right)$ which is added by a phase ramp $\left(\Delta \phi_{B i}(\xi, \eta)\right)$. Step by step means that: First we figure out how large the shift is and in which direction ( $\mathrm{x}, \mathrm{y}$ or both). Then a suitable phase ramp is generated. This phase ramp is complex multiplied by the hologram. Propagating this new hologram leads to a shifted reconstructed image. For a rotation between master and test object the image will be shifted as described. Doing so the hologram can be rotated about the necessary angle $\alpha$. At the end the object will be shifted back to its original position [27]. Usually these steps are implemented in an analysing tool.

To illustrate how a shift effects the state difference measurement of our tool we introduce a shift in x-direction into the hologram of the worn tool. Figure 6(a) illustrates a state difference where the worn tool was shifted while Figure 6(b) shows a corrected state difference. For the correction Eq. (9) was applied.

\section{COMPARISON WITH A STANDARD MEASURING TECHNIQUE}

To validate the experiments we measured the worn tool with the help of a confocal microscope (VK9700) from Keyence corp. which delivers height information of the tool including the scratches. Figure 7(a) shows a section of the state difference generated by the holographic set-up while Figure $7(b)$ shows the same section of the worn tool measured with a confocal microscope. To compare the two techniques we use a profile cut in the same area in both images. The techniques are based on different analysis tools. Therefore, the position of the profile measurement is not exactly the same. The red line in Figure 7(a) and (b) points out the cutting line for the profile section which can be seen in Figure (c) and (d). Figure 7(c) illustrates the profile of the state difference measured with the holographic set-up while Figure $7(\mathrm{~d})$ displays the comparison profile generated from the initial and worn state
FIG. 7 a) State difference of the object; b) Wear of the test object measured with a confocal microscope; c), d) Height profile of the wear along the red line in a) b) respectively.

data measured with a confocal microscope.

The comparison of the two techniques shows minor mismatches which can by explained by the measuring process and the analysis. Firstly, the confocal microscope has a nearly three times better resolution than the used holographic setup. Hence, the data coming from the confocal microscope are more detailed. Secondly, the viewing angles on the specimen of both set-ups are different. This leads to a mismatch within the $3 \mathrm{D}$ point clouds which can be recognised as a difference in the position and sectioning. Thirdly, the profiles have different zero lines and slightly different section positions which are the result of the analysis process of each of the different techniques. Fourthly, holography is a speckle based technique. Therefore, the results tend to be noisier compared to the one from the microscope as a matter of principle.

The analysis of the profiles leads to a depth of 5 to $20 \mu \mathrm{m}$ for the scratches within the investigated area. As mentioned above the synthetic wavelength of $\Lambda=123.46 \mu \mathrm{m}$ assures a range of unambiguity of $\Delta h=66.23 \mu \mathrm{m}$. Hence, the measured depth of the observed profile is therefore unambiguously determined.

\section{CONCLUSION AND OUTLOOK}

Comparative digital holography is adaptable as an inline technique measuring wear in the micro range. In contrast, using techniques like confocal microscopy wear monitoring requires removing of the tool from the micro deep drawing machine due to the limited accessibility.

Comparing the measurement time of the used techniques, the holographic method has a measurement time less than $1 \mathrm{sec}$ per hologram and the confocal microscope needs several minutes per image for the given measuring condition mentioned above. Therefore, a holographic set-up will considerably reduce the downtime of the micro deep drawing machine.

Considering the disparity in the capturing methods, the results of the comparative digital holography without a SLM 
are in good agreement with confocal microscopy. All defects within the observed range were detected.

We demonstrated that it is possible to scale the comparative digital holographic set-up down from several decimetres to several centimetres. But for the integration into the micro deep drawing machine further steps like increasing the aperture of the set-up by e.g. a lens have to be carry out. Furthermore, the accessibility to the tool must be ensured.

One benefit of comparative digital holography which was not taken into account yet is the state difference map itself. As mentioned in the introduction it is planned to introduce friction reducing micro structures upon the surface of the tool. Regarding only the phase difference of the worn tool or the result from the confocal microscope one can not say for sure if the detected structures belong to the friction reducing micro structures or if they are real wear. Even if one compares the image of the initial state it can not be ensured that there is no wear upon the friction reducing micro structures. Generation of a state difference map show only the deviations between the two states. As a result, even if there are friction reducing micro structures upon the surface only the wear will be detected.

Our next steps to realize a measurement system capable of inline quality control in an industrial environment will be:

a) Increasing the lateral resolution by introducing a long distance microscope,

b) Implementing the spatial light modulator (SLM) into the set-up and

c) Implementing the single shot measurement.

If the planned steps are successful one can say that the specific advantages of CDH - fast measurement, electro-optical alignment of initial state to worn state and adaptability to difficult boundary conditions (ambient light, vibrations, limited access) - make this technique to an interesting method for the increasing demands in industrial manufacturing especially for the mass production of micro parts where degradation of the tools result in large numbers of defective goods. An adequate quality control not only investigates the produced parts but is capable of testing the used tools during the manufacturing process and to interrupt this process before defective parts are produced. Up to now no in-line tool control in a mass micro parts production process exists. Therefore the next step will be to introduce and to qualify comparative digital holography for this goal. A first step will be the introduction of $\mathrm{CDH}$ into a demonstration process chain which is developed in the Collaborative Research Centre SFB 747 - Micro Cold Forming.

\section{ACKNOWLEDGEMENTS}

The authors thank the Deutsche Forschungsgemeinschaft (DFG) for supporting the subproject B3 (Werkzeuglebensdauer) in the Collaborative Research Centre SFB 747, Micro Cold Forming (Mikrokaltumformen) and C. Falldorf, M. Agour, T. Meeser, H. Wielage (BIAS), S. Twardy (LFM, Bremen) and T. Baumbach (bwm, Bremen), D. von Luepke (medatixx, Bamberg) for exciting discussions and support.

\section{References}

[1] N. Tiesler, and U. Engel, "Microforming Effects of Miniaturisation," in Proceedings of 8th International Conference on Metal Forming, 355-360 (Akademia Gíniczo-Hutnicza, Rotterdam, 2000).

[2] F. Vollertsen, and Z. Hu, "Tribological Size Effects in Sheet Metal Forming Measured by a Strip Drawing Test," CIRP Ann. 55, 291-294 (2006).

[3] E. Brinksmeier, 0. Riemer, and S. Twardy, "Tribological behavior of micro structured surfaces for micro forming tools," Int. J. Mach. Tool. Manu. 50, 425-430 (2010).

[4] F. Vollertsen, G. Habedank, and K. Partes, "Maßggeschneiderte Tribologie durch Laseroberflächenbehandlung," Materialwiss. Werkst. 39, 88-92 (2008).

[5] D. J. Whitehouse, "Handbook of Surface and Nanometrology," (CRC Press Taylor at Francis Group, Boca Raton London New York, 2011).

[6] J. Hoffmann, "Tastsysteme für die Mikro und Nanomesstechnik Nanometeraufgelöste Oberflächenerfassung," Tech. Mess. 75, 311-317 (2008).

[7] R. B. Bergmann, and E. Zabler, “Methoden der zerstörungsfreien Prüfung"' in Handbuch der Mess- und Automatisierungstechnik in der Produktion, H.-J. Gevatter, and U. Grünhaupt, eds., 363-410 (Springer, Berlin, 2006).

[8] F. Härtig, U. Neuschaefer-Rube, "Rückführbare Koordinatenmesstechnik für Mikrobauteile - Grundlagen und Beispiele," Tech. Mess. 75(5), 318-326 (2008).

[9] G. Binnig, C. F. Quate, and C. Gerber, "Atomic force microscope," Phys. Rev. Lett. 56, 930-933 (1986).

[10] T. Kleine-Besten, S. Loheide, U. Brand, S. Bütefisch, S. Büttgenbach, and A. Schlachetzki, "Miniaturisierter 3D-Tastsensor für die Metrologie an Mikrostrukturen," Tech. Mess. 12/99, 493-495 (1999).

[11] A. Krenn, "Optische Messverfahren für reproduzierbare Nanometer-Genauigkeit - mehr Scharfblick mit Fokus-Variation," Mikroproduktion 1(1), 51-53 (2007).

[12] R. Danzl, and F. Helmli, "Three-dimensional reconstruction of surfaces with steep slopes using an optical measurement system based on a colour focus sensor," in Proceeding of the 6th euspen International Conference 516-519 (euspen, Baden, 2006).

[13] J. C. Wyant, "White light interferometry," Proc. SPIE 4737, 98-107 (2002).

[14] http://www.keyence.de/products/vision/microscope/vk9700/ vk9700.php (09.06.2010).

[15] Z. Hu, S. Huferath-von Luepke, C. v. Kopylow, and F. Vollertsen, "Characteristics of wear behavior of micro deep drawing tools," AMPT 2010, 335-340 (2010).

[16] T. Bothe, W. Li, C. Kopylow, and W. Jüptner, "High-resolution 3D shape measurement on specular surfaces by fringe reflection," Proc. SPIE Int. Soc. Opt. Eng. 5457, 411-422 (2004).

[17] W. J. Bates, "A wavefront shearing interferometer," P. Phys. Soc. 59(6), 940 (1947).

[18] G. Pedrini, W. Osten, and Y. Zhang, "Wave-front reconstruction from a sequence of interferograms recorded at different planes," Opt. Lett. 30(8), 833-835 (2005).

[19] U. Schnars, and W. Jüptner, Digital Holography (Springer, Berlin Heidelberg New York, 2005).

[20] R. B. Bergmann, T. Bothe, C. Falldorf, P. Huke, M. Kalms, and C. von Kopylow, "Optical Metrology and Optical Non-Destructive 
Testing from the Perspective of Object Characteristics," Proc. SPIE Interferometry XV: Applications 7791, 1-15 (2010).

[21] M. Agour, P. Huke, C. v. Kopylow, and C. Falldorf, "Shape measurement by means of phase retrieval using a spatial light modulator," International Conference on Advanced Phase Measurement Methods in Optics and Imaging, AIP Conference Proceedings 1236, 265-270, (American Institute of Physics, Ascona, 2010).

[22] C. Falldorf, C. von Kopylow, and W. Jüptner, "Digitale Holografie an reflektierenden Mikrosystemen," Tech. Mess. 73, 257-265 (2006).

[23] T. Hansel, G. Steinmeyer, R. Grunwald, C. Falldorf, J. Bonitz, C. Kaufmann, V. Kebbel, and U. Griebner, "Synthesized femtosecond laser pulse source for two-wavelength contouring with simultaneously recorded digital holograms," Opt. Express 17(4), 2686-2695 (2009).

[24] W. Osten, T. Baumbach, and W. Jüptner, "Comparative digital holography," Opt. Lett. 27, 1764-1766 (2002).
[25] T. Baumbach, W. Osten, C. v. Kopylow, and W. Jüptner, "Remote metrology by comparative digital holography," Appl. Optics 45, 925-934 (2006).

[26] J. Goodman, Introduction to Fourier Optics (Roberts at Company, Greenwood Village, 2005).

[27] S. Huferath-von Luepke, T. Baumbach, E. Kolenovic, C. Falldorf, and C. v. Kopylow, "Digital Alignment of a reconstructed Hologram for Measurement of Deterioration of Tools," in Proceedings to 36th International MATADOR Conference, 331-334 (The University of Manchester, Manchester, 2010).

[28] R. B. Blackman, and J. W. Tukey, The measurement of power spectra (Dover Publications, New York, 1958).

[29] W. Hackbusch, H. Schwarz, and E. Zeidler, Teubner-Taschenbuch der Mathematik (B. G. Teubner, Stuttgart, 1996). 